\title{
Fractionnement Géochimique Des Eléments Traces Métalliques (Etm) Dans Les Sédiments Du Delta De L'ouémé Au Bénin
}

\author{
Rodrigue A.M.A. Adechina, Doctorant en Hydrologie et Gestion \\ Intégrée des Ressources en Eau \\ Nelly C. Kelome, Enseignant-Chercheur \\ Waris Chouti, Enseignant-Chercheur \\ Jéchonias B. Hounkpe, Doctorant \\ Nambinina R. F. Randriana, Enseignant-Chercheur \\ Université d'Abomey-Calavi, Bénin, \\ Université d'Antananarivo, Madagascar
}

Doi: 10.19044/esj.2018.v14n9p358 URL:http://dx.doi.org/10.19044/esj.2018.v14n9p358

\begin{abstract}
Due to the inherent damages of trace elements to living organisms, the amount of bio available metal is considered to be a quantity that conditions the becoming of aquatic ecosystems. The aim of this work is to evaluate the proportion of $\mathrm{Pb}, \mathrm{Cu}$ and $\mathrm{Cd}$ that are biologically available in the superficial sediments of the Ouémé estuary as well as the influence of environmental factors on the becoming of these metals. Fractionation of the metals was performed according to the sequential extraction method and their measurement was done by inductively coupled plasma mass spectrometer (ICP-MS). Physicochemical parameters evaluated are: granulometry, $\mathrm{pH}$, cation exchange capacity (CEC), organic carbon (Corg), iron, aluminum and calcium oxides $\left(\mathrm{Fe}_{2} \mathrm{O}_{3}, \mathrm{Al}_{2} \mathrm{O}_{3}\right.$ and $\left.\mathrm{CaO}\right)$. Risk indices related to metal fractionation were evaluated followed by statistical processing in $\mathrm{R}$ 3.3.2. It appears that: $39.44 \%$ of the $\mathrm{Cd}$ are preferentially bound to the exchangeable fraction, $37.25 \%$ of the $\mathrm{Pb}$ are for the reducible fraction and $47.47 \%$ of the $\mathrm{Cu}$ for the oxidizable fraction. Risk levels are in the low to very high range. The richness of materials is revealed as a factor limiting the bioavailability of metals while $\mathrm{CaO}$ facilitates the bioavailability in high risk sites. Liquid and solid wastes from the Dantokpa market are the potential sources of metals.
\end{abstract}

Keywords: Ouémé estuary, sediments, ETM, fractionation 


\section{Résumé}

En raison des dommages inhérents aux Eléments traces Métalliques (ETM) pour les organismes vivants, la quantité du métal biodisponible est considérée comme une grandeur qui conditionne le devenir des écosystèmes aquatiques. Le but du travail est d'évaluer la proportion $\mathrm{du} \mathrm{Pb}, \mathrm{Cu}$ et $\mathrm{Cd}$ biodiponible dans les sédiments superficiels de l'estuaire de l'Ouémé ainsi que l'influence des facteurs environnementaux sur le devenir de ces métaux. Le fractionnement des métaux a été réalisé suivant la méthode d'extraction séquentielle et leur dosage a été fait au spectromètre de masse par plasma à ouplage inductif (ICP-MS). Des paramètres physico-chimiques déterminés sont : la granulométrie, le $\mathrm{pH}$, la Capacité d'échange cationique (CEC), le Carbone organique (Corg), les oxydes de fer, d'aluminium et de calcium $\left(\mathrm{Fe}_{2} \mathrm{O}_{3}, \mathrm{Al}_{2} \mathrm{O}_{3}\right.$ et $\left.\mathrm{CaO}\right)$. Des indices de risque liés au fractionnement des métaux ont été évalués suivi du traitement statistique au logiciel R 3.3.2. Il ressort que : $39,44 \%$ du $\mathrm{Cd}$ sont préférentiellement liés à la fraction échangeable, $37,25 \%$ du $\mathrm{Pb}$ le sont pour la fraction réductible et $47,47 \%$ du $\mathrm{Cu}$ pour la fraction oxydable. Les niveaux de risque sont dans la fourchette de faible à très haut degré. La richesse en matières est révélée comme un facteur limitant la biodisponibilité des métaux tandis que le $\mathrm{CaO}$ facilite la dites biodisponibilité dans les sites à haut risque. Les déchets liquides et solides du marché de Dantokpa sont les potentielles sources de métaux.

Mots-clefs: Estuaire de l'Ouémé, sédiments, ETM, fractionnement

\section{Introduction}

Les activités anthropiques telles que l'agriculture, l'industrie minière, la fonderie,... sont de potentielles sources en Eléments Traces Métalliques (ETM) dans les cours d'eau (Othmani et al., 2015 ; Alharbi et Sorogy, 2017). Parmi ces Eléments Traces Métalliques, le cadmium, le plomb, le mercure et le nickel sont classés sur la liste des substances prioritaires de la Directive 2008/105/CE (Cadre sur l'Eau) et font l'objet de préoccupation environnementale majeure en raison de leur toxicité, leur propriété non biodégradable, leur mobilité et leur biodisponibilité (Olujimi et al., 2015). Dans les organismes vivants, ces ETM peuvent entraîner des anomalies morphologiques, des troubles neurophysiologiques, affecter les activités enzymatiques et hormonales (Okoro et al., 2012 ; Wang et al., 2017). Ainsi, pour attirer l'attention des gouvernements et des chercheurs, Hann qualifia ces métaux de «bombe à retardement chimique » (Zheng et al., 2015).

L'ensemble des travaux de recherche menés jusqu'à ce jour ont prouvé la vulnérabilité de l'estuaire de l'Ouémé vis-à-vis des ETM (Kaki et al., 2011; Youssao et al., 2011 ; Hounkpatin et al., 2012). Les résidus des produits 
agrochimiques utilisés pour la culture du coton, les eaux usées résidentielles, les déversements de produits pétroliers provenant du Nigéria... sont les probables sources des ETM (Assogba, 2014). Cependant, Zhou (2009) a fait remarquer que la concentration en métal total peut être élevée alors que la quantité de métal biodisponible est faible. En plus, Daldoul et al., (2015) a renseigné que seules les espèces métalliques labiles (soluble, échangeables) sont disponibles et peuvent être plus toxiques. Certes, les sédiments de l'estuaire de l'Ouémé sont contaminés en ETM mais leur distribution géochimique n'est pas connue. La présente étude vise à évaluer les niveaux de risque potentiel de pollution en $\mathrm{Pb}, \mathrm{Cu}$ et $\mathrm{Cd}$ des sédiments superficiels ainsi que le rôle des conditions physico-chimiques sur la libération de ces métaux dans l'eau. Elle renseigne si, pour de fortes teneurs totales en ETM, les ressources biologiques sont effectivement exposées.

\section{Approche méthodologique \\ Milieu d'étude}

L'estuaire de l'Ouémé est localisé dans la partie méridionale du Bénin, un des pays ouest-africains. Cet estuaire est intégré au bassin sédimentaire côtier du Bénin (Laibi, 2011) (Figure 1). Les formations géologiques sont constituées de dépôts quaternaires qui reposent sur une épaisse couche de grès, d'argiles, du calcaire... dont les âges remontent jusqu'au Cénomanien. La lithologie des dépôts quaternaires est composée d'argiles, de sables, de vases, de tourbe... Le climat est du type subéquatorial avec des températures moyennes de l'air d'environ $28,33^{\circ} \mathrm{C}$ à la grande saison sèche et $27,46^{\circ} \mathrm{C}$ à la grande saison pluvieuse. Du point de vue hydrographique, le fleuve Ouémé est le principal cours d'eau de l'estuaire avec ses affluents que sont l'Okpara et le Zou. La moyenne mensuelle des débits de 1970 à 2010 montre un débit minimal de $5,49 \mathrm{~m}^{3} / \mathrm{s}$ en mars et un maximum de $621,42 \mathrm{~m}^{3} / \mathrm{s}$ en septembre. En hautes eaux (juillet, août, septembre, octobre) des débordements se font vers la rivière Sô et la salinité dans le cours d'eau est d'environ 0,1 \% (Moniod, 1973). A l'étiage (décembre à mai), la salinité est proche de $2 \%$ à l'embouchure de la Sô et ne dépasse pas $1 \%$ pour l'Ouémé. C'est pendant cette dernière période, que se produit la remontée des eaux lagunaires dans le fleuve (Le Barbé et al., 1993). 


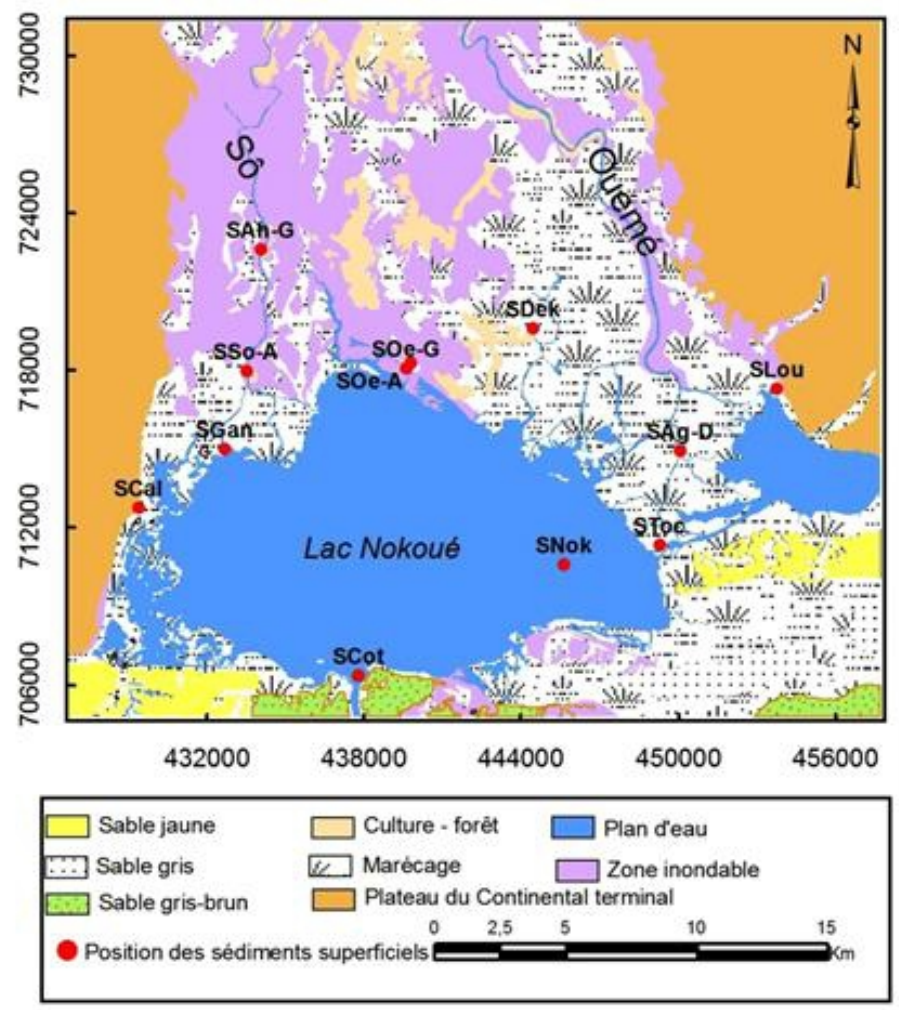

Figure 1. Carte montrant le delta de l'Ouémé et les points d'échantillonnage

\section{Echantillonnage et méthodes analytiques}

Une campagne de terrain a été organisée du 10 au 13 août 2015. A cet effet, douze échantillons de sédiments superficiels ont été prélevés au moyen d'une benne de Eckman dans douze points (Figure 1). Les sites d'échantillonnage sont: Toché (SToc), Nokoué (SNok), Louho (SLou), Aguégué-Donoukpa (SAg-D), Dékanmè (SDek), Ouédo-Aguékon (SOe-A), Ouédo-Gbadji (SOe-G), Ahomey-Glon (SAh-G), Ganvié (SGan), débarcadère Sô-Ava (SSo-A), embarcadère Calavi (SCal), débouché du chenal Cotonou (SCot). Les sédiments ont été ensuite emballés dans les sachets en plastique puis étiquetés. Tous les échantillons ont été ensuite acheminés au laboratoire le plus rapidement possible pour être conservés dans un réfrigérateur à $3-4^{\circ} \mathrm{C}$. Les paramètres physico-chimiques ont été évalués au Laboratoire de Chimie Minérale de l'Ecole Supérieure Polytechnique d'Antananarivo (Madagascar). Pour l'étude quantitative des classes dimensionnelles des particules, $200 \mathrm{~g}$ de sédiments secs ont été prélevés, lavés puis tamisés par une série de neuf tamis du module AFNOR NFX 11504. La détermination du pH est basée sur le mode opératoire classique. La méthode d'évaluation de la CEC est celle de trichlorure de cobaltihexammine spécifiée par ISO 23470 : 2007. Les pourcentages en Corg des sédiments ont été connus suivant les Lignes 
directrices concernant les travaux analytiques en chimie DR-12-CSA-01 du Centre d'expertise en analyse environnementale du Québec. La détermination de la teneur des éléments majeurs est basée sur la méthode volumétrique par titrage à l'EDTA. Le protocole de Tessier et al. (1979) modifié par Schultz et al. (1998) et Bouzidi et al. (2015) a été utilisé pour le fractionnement des métaux dans $1 \mathrm{~g}$ de sédiment brut (Tableau.1). Le dosage a été fait à l'ICPMS de 1'Office des Mines Nationales et des Industries Stratégiques (OMNIS / Madagascar). Pour la précision et l'exactitude des mesures à l'ICP-MS, des matériaux de référence standard (Perkin Elmer 18 - 195 JB, N9300233) ont été utilisés, trois standards ont été réalisés et un essai témoin a été effectué en parallèle sur un échantillon de d'eau purifiée HNO3 5\%. L'évaluation des risques environnementaux a été basée sur le Code d'Evaluation des Risque "Risk Assessment Code" (RAC) exprimé par :

$$
R A C=\% \text { H.sol. }+\% \text { Ech. }+\% \text { A.sol } \text {. }
$$

Avec \% H.sol., le pourcentage de la fraction hydrosoluble, \% Ech., le pourcentage de la fraction échangeable et \% A.sol., le pourcentage de la fraction acido-soluble (Perin et al., 1985). Ce sont ces trois fractions qui contribuent à l'équilibre dans la phase aqueuse et sont donc rapidement biodiponibles.

Les coefficients de corrélation de Pearson ont été utilisés pour étalier les relations entre les paramètres environnementaux avec un niveau de confiance de $95 \%$ au logiciel R 3.3.2. Une analyse en composante principale (ACP) est utilisée pour identifier les principales sources d'émissions des polluants et les paramètres qui influencent la biodisponibilité des ETM.

Tableau 1. Protocole de fractionnement de ETM

\begin{tabular}{|c|c|c|}
\hline Fractions extraites & Réactifs & Condition \\
\hline Hydrosoluble & $15 \mathrm{ml}$ eau ultrapure & Agitation continue $; 20{ }^{\circ} \mathrm{C} ; 1 \mathrm{~h}$ \\
\hline Echangeable & $8 \mathrm{ml} \mathrm{MgCl} 2(1 \mathrm{M})$ & $\begin{array}{c}\text { Agitation continue } ; \mathrm{pH} 7 ; 20 \\
{ }^{\circ} \mathrm{C} ; 1 \mathrm{~h}\end{array}$ \\
\hline Oxydable & $15 \mathrm{ml} \mathrm{NaCLO}(5-6 \%)$ & $\begin{array}{c}\text { Agitation intermittente } ; \mathrm{pH} \\
7,5 ; 96{ }^{\circ} \mathrm{C} ; 30 \mathrm{mn} \times 2\end{array}$ \\
\hline Acido-soluble & $8 \mathrm{ml} \mathrm{CH} 3 \mathrm{COONa}(1 \mathrm{M}) / \mathrm{CH} 3 \mathrm{COOH})$ & $\begin{array}{c}\text { Agitation continue } ; \mathrm{pH} 5 ; 20 \\
{ }^{\circ} \mathrm{C} ; 5 \mathrm{~h}\end{array}$ \\
\hline $\begin{array}{c}\text { Réductible }(\mathrm{Fe}- \\
\text { Mn })\end{array}$ & $20 \mathrm{ml} \mathrm{NH} 2 \mathrm{OH} . \mathrm{HCl}(0.5 \mathrm{M})+\mathrm{CH} 3 \mathrm{COOH}$ \\
$(25 \%)$ & $\begin{array}{c}\text { Agitation intermittente } ; 95{ }^{\circ} \mathrm{C} ; \\
5 \mathrm{~h}\end{array}$ \\
\hline Résiduelle & $40 \% \mathrm{HF} / 60 \% \mathrm{HClO} 4 / 38 \% \mathrm{HCl} / 65 \% \mathrm{HNO} 3$ & A sec \\
\hline
\end{tabular}

\section{Résultats et discussion}

\section{Caractérisation physico-chimique des sédiments superficiels}

$\mathrm{Au}$ nombre des faciès constituant les sédiments analysés, il est distingué les sables moyens à grossiers dans lesquels les teneurs en sable (2 $0,065 \mathrm{~mm}$ ) varient entre $86,33 \%$ et $91,1 \%$. Ces sédiments caractérisent les échantillons prélevés à Ahomey-Gblon, à Sô-Ava. Les sables à fins à moyens 
sont représentés par les sédiments des localités de Dékanmè, Ouédo-Gbadji et Ouédo-Aguékon dans lesquels les teneurs en sable varient de 92,47 à 96,8\% . Les sables moyen à fin faiblement argileux sont représentés par les sédiments prélevés dans le chenal de Cotonou, à l'embarcadère de Calavi et dans le chenal de Toché. Les proportions de sable sont comprises entre 80,93 et 90,2 $\%$. Les sables fin argileux sont prélevés dans le lac Nokoué et à AguéguéDonoukpa et contiennent respectivement $79,53 \%$ et $84,06 \%$ de sable. Par contre les sables argilo-silteux des secteurs de Ganvié et Louho en contiennent respectivement 59,53\% et 53,46\% de sable. Pour l'ensemble des douze sites, les $\mathrm{pH}$ sont quasiment neutres et varient très peu entre 5 à Louho et 6,5 à l'embouchure du chenal de Cotonou pour une moyenne de 5,50. Les CEC oscillent entre 65,25 méq/100g à Ouédo-Gbadji et 99,04 méq/100g dans les sédiments de Louho avec une moyenne de 84, 24 méq/100g. Le minimum du Corg $(0,01 \%)$ a été obtenu dans les sédiments de Ouédo-Gbadji et le maximum $(3,33 \%)$ dans les sédiments de Ganvié avec une moyenne de $0,84 \%$. Les moyennes des teneurs en $\mathrm{Al}_{2} \mathrm{O}_{3}$ et en $\mathrm{Fe}_{2} \mathrm{O}_{3}$ sont respectivement $8,14 \%$ et $3,9 \%$. La plus faible teneur en $\mathrm{Al}_{2} \mathrm{O}_{3}$ est mesurée dans les sédiments de Ouédo-Gbadji $(0,82 \%)$ et la plus forte teneur $(37 \%)$ est obtenue à SôAva. Le $\mathrm{Fe}_{2} \mathrm{O}_{3}$ est également plus concentré à Sô-Ava $(12,42 \%)$ mais sa teneur minimale est déterminée à Ahomey-Glon $(0,9 \%)$. Le $\mathrm{CaO}$ montre une variation peu significative entre 4,51 \% (Ouédo Aguékon) et 7,81\% (à Ganvié) pour une moyenne de 6,08 \% (Tableau 2). Une forte corrélation linéaire positive (coefficient de corrélation $r>0.75$ ) est remarquée entre la $\mathrm{CEC}$, la fraction inférieure à $63 \mu \mathrm{m}$ et le Corg. Quant aux éléments majeurs analysés, seul les pourcentages en $\mathrm{Al}_{2} \mathrm{O}_{3}$ et le $\mathrm{Fe}_{2} \mathrm{O}_{3}$ indiquent une forte corrélation $(\mathrm{r}=0,93)$. Le $\mathrm{pH}$ n'est corrélé à aucun de tous ces paramètres. La première établie entre le pourcentage de la fine, la CEC et le pourcentage du Corg concorde avec les résultats des travaux de Bouzidi et al. (2015) dans la région de Ain Oussera au Sud d'Alger. Cette liaison s'explique par la présence de substances humiques capables de former des complexes avec les minéraux argileux, ce qui favorise 1'augmentation de la $\mathrm{CEC}$ et donc la rétention des métaux dans les sédiments. La seconde liaison établie entre $\mathrm{Al}_{2} \mathrm{O}_{3}$ et $\mathrm{Fe}_{2} \mathrm{O}_{3}$ témoigne d'un rapport entre Fe/Al constant. Selon Othmani et al. (2015), ce rapport dans les sédiments de l'ancien district minier de Touiref (NW Tunisie), suggère une dominance de la kaolinite et du chlorite et reflète les processus d'altération qui ont lieu en amont.

Tableau 2. Paramètres physico-chimiques et teneurs en ETM des sédiments

\begin{tabular}{|c|c|c|c|c|c|c|c|c|c|c|}
\hline Sites & $\mathrm{pH}$ & $\%$ Fine & $\mathrm{CEC}$ & $\%$ Corg & $\% \mathrm{Fe}_{2} \mathrm{O}_{3}$ & $\% \mathrm{Al}_{2} \mathrm{O}_{3}$ & $\% \mathrm{CaO}$ & $\mathrm{Cu}$ & $\mathrm{Pb}$ & $\mathrm{Cd}$ \\
\hline SNok & 5,3 & 17,9 & 94,1 & 0,96 & 3,4 & 2,96 & 6,77 & 21 & 19,1 & 1,35 \\
\hline SToc & 5,1 & 10,1 & 89,6 & 0,83 & 2,97 & 1,89 & 6,77 & 15 & 32,5 & 3,9 \\
\hline SLou & 5 & 45,7 & 99 & 1,26 & 4,5 & 12,1 & 5,25 & 11,1 & 4,9 & 0,001 \\
\hline SAg-D & 5,5 & 12,8 & 93,6 & 0,95 & 2,97 & 2,37 & 5,51 & 16 & 8,6 & 2,2 \\
\hline SDek & 5,6 & 2,1 & 79,8 & 0,28 & 2,22 & 1,18 & 5,21 & 21,33 & 0,8 & 0 \\
\hline
\end{tabular}




\begin{tabular}{|c|c|c|c|c|c|c|c|c|c|c|}
\hline SOe-A & 5,1 & 7 & 82,4 & 0,8 & 2,78 & 1,42 & 4,51 & 34,86 & 0,96 & 0,003 \\
\hline SOe-G & 5,4 & 1,6 & 65,2 & 0,01 & 0,9 & 0,82 & 5 & 14,6 & 0,38 & 0 \\
\hline SAh-G & 6 & 1,6 & 65,6 & 0,14 & 1,48 & 0,9 & 5,73 & 14,4 & 7,1 & 1,6 \\
\hline SGan & 5,3 & 36,5 & 97,5 & 3,33 & 4,45 & 5,4 & 7,8 & 149 & 22,5 & 2,6 \\
\hline SSo-A & 5,1 & 5,2 & 80 & 0,5 & 12,4 & 37 & 6,7 & 32,9 & 44,3 & 0,9 \\
\hline SCal & 5,3 & 1,8 & 77,4 & 0,2 & 1,85 & 1,2 & 6,2 & 13,3 & 33,9 & 0,003 \\
\hline SCot & 6,5 & 7,8 & 86,7 & 0,8 & 6,8 & 30,5 & 7,5 & 51,5 & 104 & 4,6 \\
\hline
\end{tabular}

\section{Fractionnement des ETM dans les sédiments}

Les pourcentages de récupération d'extraction séquentielle expriment le rapport en pourcent entre la teneur totale du métal dans le sédiment brut et la teneur totale dans les six fractions. Ces pourcentages varient respectivement de 88,75 à $99,67 \%$; de 88,15 à $101,025 \%$ et de 87,5 à $101 \%$ pour le $\mathrm{Cu}$, le $\mathrm{Pb}$ et le $\mathrm{Cd}$. Les pourcentages substantiels de $\mathrm{Cu}$ sont enregistrés dans les fractions oxydables, réductibles et acido-solubles. Le $\mathrm{Cu}$ est représenté à des pourcentages variant de 27,81 \% (SSo-A) à 63,14\% (SDek) dans la fraction oxydable, de 6,63 \% (SCal) à 28,91 \% (SDek) dans la fraction acido soluble et de $0,54 \%$ (SAh-G) à 36,35\% (SSo-A) dans la fraction réductible. Excepté les sédiments de Louho, Ahomey-Gblon dans lesquels le Cd est fortement lié à la fraction réductible et Ganvié où il est plus concentré dans la fraction organique, le $\mathrm{Cd}$ admet une forte affinité pour la fraction échangeable dans l'ensemble des sites. Ses proportions oxillent de 5,71\% (SAh-G) à 64,28\% (SCal) dans la fraction échangeable, de 0,007 \% (SCot) à 47,48\% (SAh-G) dans la fraction réductible, de 0,22 \% (SAg-G) à 47,14 \% (SNok) dans la fraction acido-soluble et de 0,04\% (SAg-D) à 39,73\% (SGan) dans la fraction organique. La répartition $\mathrm{du} \mathrm{Pb}$ montre une similitude avec celle du $\mathrm{Cd}$ mais avec une affinité prononcée pour la fraction réductible. Sa répartition varie de $0,21 \%$ (SLou) à 56,52 \% (SCal) dans la fraction échangeable, de 24,54\%( SNok) à 68,25\% (SLou) dans la fraction réductible et de 0,41 \% (SLou) à $28,03 \%$ (SGan) dans la fraction acido-soluble (Figure 2). En général, l'ordre décroissant de l'affinité de chacun des trois métaux vis-à-vis des six phases géochimiques est établi comme suit : $\mathrm{Cu}$ : oxydable $(47,47 \%)>$ acido-soluble $(19,98 \%)>$ réductible $(15,97 \%)>$ hydrosoluble $(7,77 \%)>$ résiduelle $(5,98$ $\%)>$ échangeable $(5,41 \%)$. Cd : échangeable $(39,44 \%)>$ acido-soluble $(24,59 \%)>$ réductible $(14,87 \%)>$ résiduelle $(13,66 \%)>$ hydrosoluble $(9,83$ $\%)>$ oxydable $(9,67 \%)$. $\mathrm{Pb}:$ réductible $(37,25 \%)>$ échangeable $(30,07 \%)>$ acido-soluble $(13,88 \%)>$ résiduelle $(9,31 \%)>$ oxydable $(8,64 \%)$ hydrosoluble $(5,03 \%)$.

L'affinité des métaux aux phases géochimiques montre que trois phases majeures concentrent le $\mathrm{Pb}$ et le $\mathrm{Cd}$. Il s'agit des phases échangeables, réductibles et acido-solubles. Dans le cas du Cd, Chen et al. (2014) en Suining (Chine) puis Li et al. (2015) dans le parc olympique de Beijing (Chine) pensent que les propriétés chimiques plus actives des ions $\mathrm{Cd}$ les rendent 
facilement échangeables que les autres. Selon Sundaray et al. (2011) dans le bassin estuarien de Mahanadi (Inde), la similitude du rayon ionique du $\mathrm{Cd}$ $(0.97 \AA)$ et du Ca $(0.99 \AA)$ favorise la co-précipitation des carbonates $\mathrm{Cd}$ et son incorporation dans le réseau de calcite pour former des solutions solides de $\mathrm{Cd} \alpha \mathrm{Ca} 1-\alpha \mathrm{CO} 3$. Cowan et al. (1991) a déjà remarqué une diminution significative (jusqu'à 40\% à $\mathrm{pH} 7,2$ ) de l'adsorption du $\mathrm{Cd}$ par des oxydes amorphes Fe en présence de Ca. Par ailleurs, Okoro et al. (2012) ont rapporté que les métaux ont tendance à être plus biodisponibles à faible salinité. Bien que l'estuaire de l'Ouémé, soit sujet aux remontées périodiques de la marée (Le Barbé et al., 1993), le taux de sel n'étant sans doute pas significatif pendant l'accumulation des sédiments a influencé la distribution $\mathrm{du} \mathrm{Cd}$ et $\mathrm{du}$ $\mathrm{Pb}$ en facilitant leur liaison dans les biodiponibles. Quant à l'affinité du $\mathrm{Pb}$ à la fraction acido-soluble, Bouzidi et al. (2015) ont attesté la présence de cérusite dans laquelle le $\mathrm{Pb}$ est bio accessible. L'affinité $\mathrm{du} \mathrm{Pb}$ pour la fraction acido-soluble dans les sédiments de l'Ouémé peut s'expliquer par ce type de liaison, vu la forte corrélation reconnue entre les fractions biodiponibles du $\mathrm{Pb}$ et du $\mathrm{Cd}$ et le CaO. Néanmoins, El Azhari et al. (2016) a montré que la minéralogique des sédiments du fleuve Moulouya (Maroc) ne contrôlent pas entièrement la distribution de $\mathrm{Pb}$. Helali et al. (2017) dans le fleuve de Mejerda (golfe de Tunis) ont insisté sur l'influence de la saisonnalité et de la sédimentation sur le fractionnement des métaux. Pour ces derniers, l'étroite liaison $\mathrm{du} \mathrm{Pb}$ aux oxyhydroxydes et aux carbonates est limitée aux matières en suspension et la fraction échangeable est observée pendant la saison sèche. Zhao et al. (2012) ont plutôt rapporté que la formation du $\mathrm{Pb}$ réductible évoque l'influence des constantes de stabilité élevées des oxydes de $\mathrm{Pb}$. Concernant la préférence du $\mathrm{Cu}$ pour la phase organique, cette dernière s'explique par l'affinité des substances humiques pour les métaux bivalents, lesquelles représentent la matière organique la plus active dans leur complexation. Cette liaison (M.O.-métaux) qui décroit du $\mathrm{Cu}^{2+}\left(5,0\right.$ à 21,3\%), $\mathrm{Pb}^{2+}(5,6$ à 16,1\%) au $\mathrm{Zn}^{2+}(5,5$ à $13,9 \%)$ (Zhao et al., 2012) justifie le pourcentage élevé du $\mathrm{Cu}$ oxydable $(47,47 \%)$ dans cette étude. Dans une approche d'étude de la spéciation des métaux par titrage acidimétrique dans l'estuaire de Scheldt (Pays-Bas), Petit et al. [2009] ont par contre suggéré que $70 \%$ du cuivre présents dans la phase oxyhydroxyde ont co-précipité avec la gibbsite. Parallèlement, Alharbi et al. (2017) ont remarqué une association entre le $\mathrm{Cu}$, l'Al et la fraction résiduelle dans les sédiments de Al-Khobar (Arabie Saoudite). 
$\mathrm{Cu}$

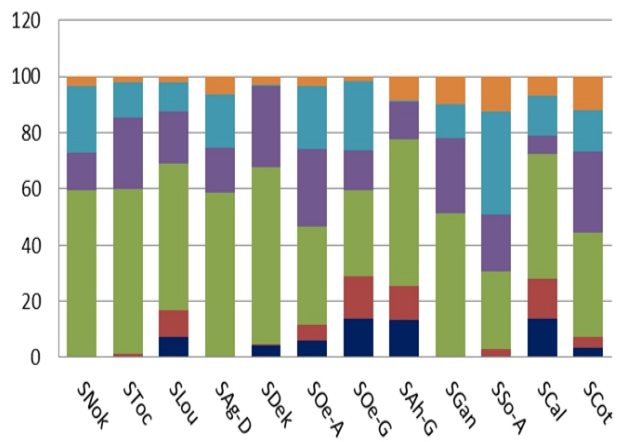

$\mathrm{Pb}$

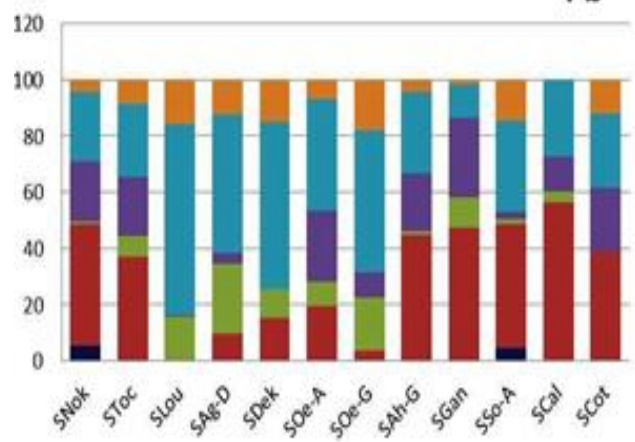

Cd

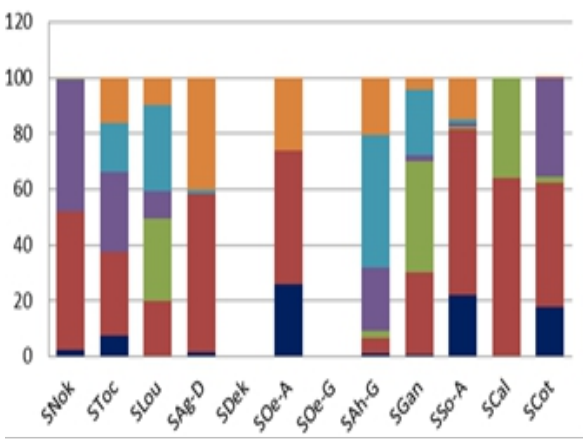

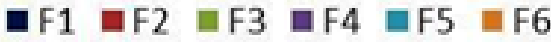

F1 : hydrosoluble ; F2 : Echangeable ;

F3 :Oxydable ;

F4 : Acido-soluble ; F5 ; Réductible ;

F6 : Résiduelle

Figure 2. Tuyaux d'orgue de fractionnement des ETM dans les différentes phases géochimiques de l'estuaire de l'Ouémé

\section{Evaluation des risques écologiques lies a la contamination}

En considérant les teneurs limites fixées qui sont de $0,11 \mathrm{ppm}$ (directive /76/464/CEE 2000) pour le cas le Cd, il est remarqué que les sédiments SNok, SToc, Ag-D, SAh-G, SGan, SSo-A et SCot sont pollués en $\mathrm{Cd}$ (Tableau 2). Concernant le $\mathrm{Pb}$, les limites sont franchises dans les sédiments SNok, SToc, SGan, SSo-A, SCal et SCot en référence aux seuils à $19 \mathrm{ppm}$. Seuls les échantillons SOe-A, SGan et SCot dépassent les limites de $33 \mathrm{ppm}$ pour le $\mathrm{Cu}$. Du point de vue écologique, le "Risk Assessment Code" (RAC) du $\mathrm{Cu}$ des sites pollués révèle un niveau de risque écologique moyen à élevé. Les sites pollués en $\mathrm{Pb}$ ont un niveau de risque catastrophique (Figure 3). Malgré que les seuils n'aient pas été franchis dans certains sites, des niveaux de risques élevés en $\mathrm{Pb}$ sont enregistrés. C'est le cas de Louho, Dékanmè, Ouédo-Gbadji, Ahomey-Glon et Calavi pour le $\mathrm{Cu}$. Ce même constat est valable sur les sites de Ouédo-Aguékon, Ahomey-Gblon pour le $\mathrm{Pb}$ puis Ouédo-Aguékon, Calavi pour le $\mathrm{Cd}$. Le RAC des sites pollués en $\mathrm{Cd}$ montre un niveau de risque écologique moyen à catastrophique. Les observations faites sur les sites non pollués et à risques élevé concordent avec les travaux de Zhou (2009) qui ont signalé que la concentration en métal total peut être élevée alors que la quantité de métal biodisponible est faible. 


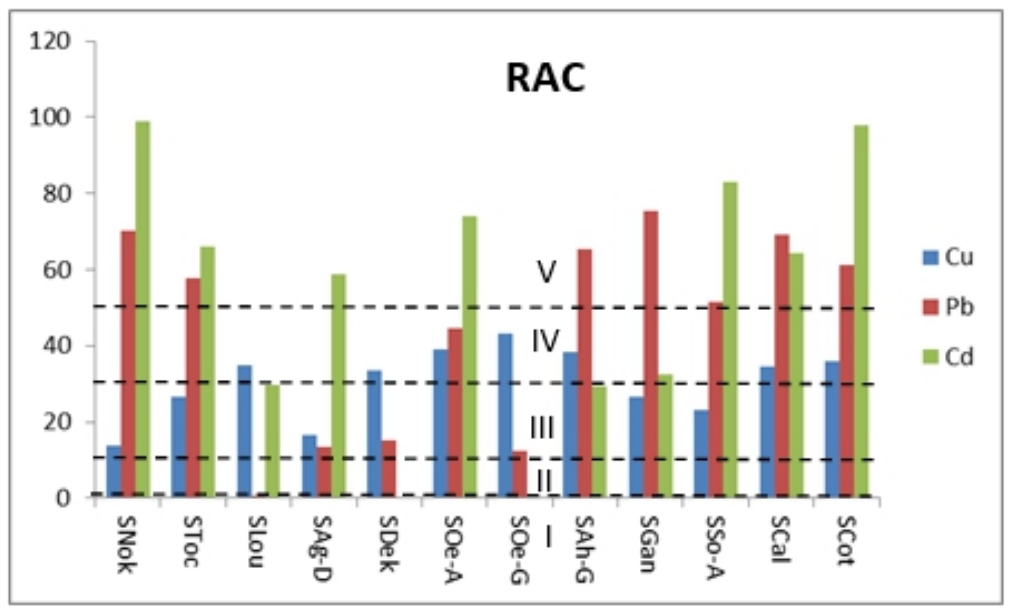

Figure 3. Variation du RAC dans les sédiments superficiels de l'estuaire de l'Ouémé. I : pas de risque $(\mathrm{RAC} \leq 1 \%)$; II : risque faible $(1<\mathrm{RAC} \leq 10 \%)$; III : risque moyen $(11<\mathrm{RAC} \leq$ $30 \%) ; \mathrm{IV}$ : risque élevé $(31<\mathrm{RAC} \leq 50 \%) ; \mathrm{V}$ : risque catastrophique $(50 \%<\mathrm{RAC})$.

\section{Evaluation des sources de contamination}

Pour la détermination des sources de contamination et les facteurs qui influencent la biodisponibilité des ETM, les teneurs de la fraction biodisponible (métalB) ainsi que les pourcentages en $\mathrm{Corg}, \mathrm{Fe}_{2} \mathrm{O}_{3}$ et $\mathrm{CaO}$ des sédiments ont été soumis à une analyse en composante principale (Figure 4). La fraction biodisponible représente la somme des fractions hydrosoluble, échangeable et acido-soluble (Perin et al., 1985 ; Zhao et al., 2012). Les trois premières dimensions expriment respectivement $49,01 \%, 17,49 \%$ et $12,25 \%$ de la variance totale. La première dimension est fortement corrélée aux fractions biodiponibles du $\mathrm{Pb}$, du $\mathrm{Cd}$ et au $\mathrm{CaO}$ (coefficients de corrélation $\mathrm{r}$ $>0.75$, p.value < 0.007). Elle traduit un apport anthropique important en $\mathrm{Pb}$, en $\mathrm{Cd}$ et est dite anthropogène (El Azhari et al., 2016). Cette dimension caractéristique des sites de l'embouchure du chenal de Cotonou, du Nokoué et de Toché montre que les sédiments de ces zones bénéficient d'un flux d'eau marine riche en $\mathrm{Ca}$ et des rejets provenant du marché de Dantokpa. L'origine des métaux des sédiments de Dékanmè, Ouédo-Gbadji, Ouédo-Aguékon qui s'oppose à cette source suggère un apport naturel important à travers le processus d'altération des roches.

Quant à la seconde dimension, elle définit une liaison forte avec le Corg $(r=0,75$, p.value < 0.006) et est caractéristique des sédiments de Ganvié. $\mathrm{Vu}$ la forte liaison établie entre le Corg, le pourcentage de la fraction fine et la $\mathrm{CEC}$, les paramètres texturaux ou des phénomènes naturels tels que la complexation des ETM avec la M.O., influencent l'accumulation des métaux dans ces sédiments. Cette dimension est qualifié «d'argileuse » (El Azhari et al., 2016). Les pourcentages en $\mathrm{Fe}_{2} \mathrm{O}_{3}$, le Cd biodisponible et les métaux des sédiments de Sô-Ava s'oppose à cette dimension. 


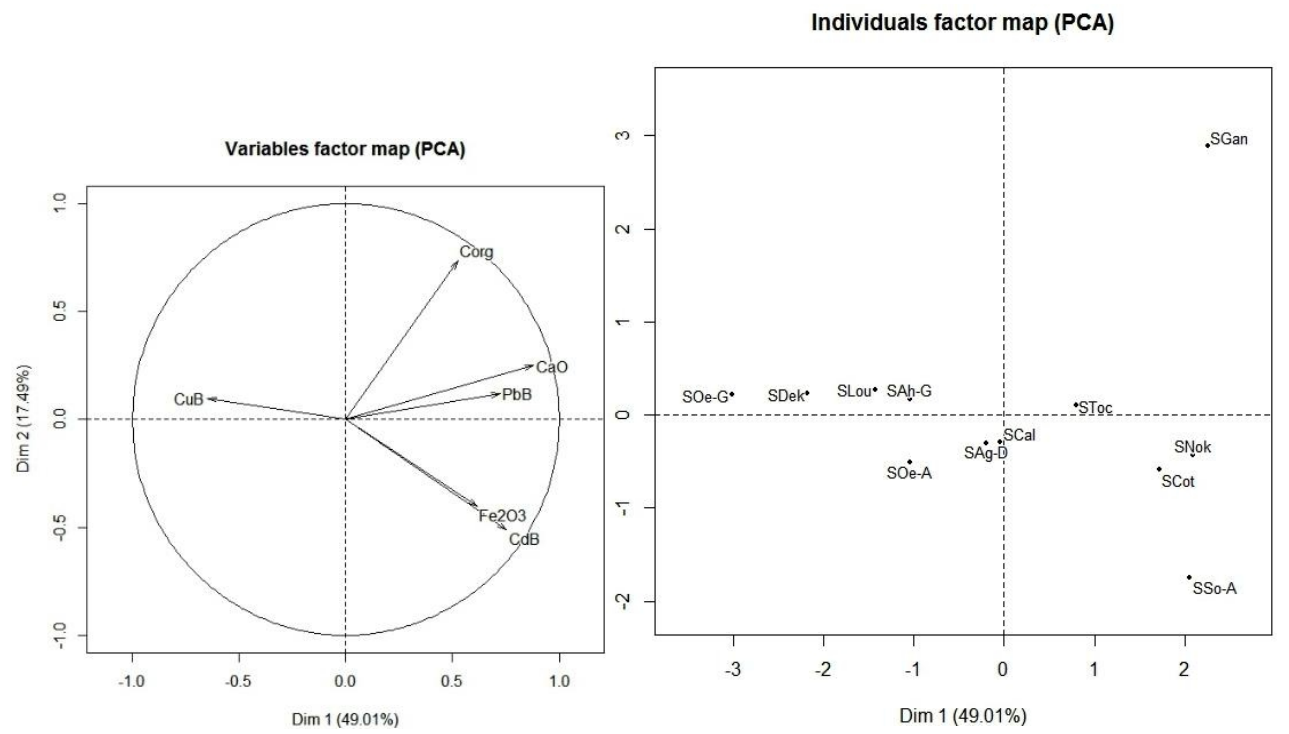

Figure 4. Diagramme factoriel des teneurs de la fraction bio disponible des métaux et des paramètres environnementaux

La troisième dimension est moyennement corrélée au $\mathrm{Pb}$ biodisponible $(\mathrm{r}=0,64$, p.value $<0.02)$. Elle est caractéristique des sites de Calavi, AhomeyGblon, Ouédo-Aguékon dont les sédiments ont sans doute bénéficié d'un apport anthropique modéré du Pb liés soit aux rejets domestiques, soit anciens déversements accidentels des produits pétroliers dans leurs débarcadères ou leur points de vente situés au voisinage de ces sites.

\section{Conclusion}

Des sédiments de l'estuaire de l'Ouémé confirment la vulnérabilité de cet écosystème à la pollution en $\mathrm{Pb}, \mathrm{Cd}$ et $\mathrm{Cu}$. $\mathrm{Le} \mathrm{Pb}$ et le $\mathrm{Cd}$ sont préférentiellement liés aux fractions échangeables, acido-solubles et réductibles des sédiments. Par contre, le $\mathrm{Cu}$ admet plus d'affinité à la fraction oxydable. Les sites de Cotonou, Toché et Nokoué qui montrent un niveau de risque élevé reflètent un apport anthropique important. Les M.O. sont révélées comme un facteur limitant la biodisponibilité des métaux tandis que le $\mathrm{CaO}$ est révélé comme le paramètre qui facilite la biodisponibilité dans les sites à haut risque. La distribution géochimique des métaux surtout du $\mathrm{Pb}$ et du $\mathrm{Cd}$ s'est avérée plus dangereuse pour les ressources biologiques. Des stratégies contribuant à la réduction des déversements de liquides acides devront être envisagées surtout aux environs de Cotonou. Car, des conditions acides sont favorables à la libération de ces métaux. Bien que la disponibilité des fractions oxydables soit limitée, un retour aux conditions réductrices peut faciliter leur mise en solution. Ces dernières conditions devront être très préoccupantes 
dans le secteur de Ganvié. Enfin, des techniques de dépollution des sédiments doivent être envisagées pour la gestion durable de l'estuaire de l'Ouémé.

\section{Remerciements}

Ce document s'inscrit dans le prolongement d'un programme de recherche appuyé par la Fondation Internationale pour la Sciences (IFS). A travers ce manuscrit, nous exprimons nos vifs remerciements à l'endroit du staff de ladite organisation.

\section{References:}

1. Alharbi, T., El-Sorogy, A. (2017). Assessment of metal contamination in coastal sediments of Al-Khobar, Jour. Afric. Ear. Scie., 129: 458-468.

2. Assogba, D. C. (2014). La production de tomates, un risque de santé pour les paysans. https://www. scidev.net/afrique-sub-saharienne.

3. Bouzidi, A., Ararem, A., Imessaoudene, D., Yabrir, B. (2015). Sequential extraction of $\mathrm{Cs}$ and $\mathrm{Sr}$ from Ain Oussera soils around EsSalam research reactor facility. Jour. Environ. Sci., 36 : 163-172.

4. CEE, 1976: Directive 76-464-CEE du 04/05/76 concernant la pollution causée par certaines substances dangereuses.

5. Centre d'expertise en analyse environnementale du Québec. Guide d'échantillonnage à des fins d'analyse environnementale, Ministère du Développement durable, de l'Environnement et des Parcs du Québec, Edition courante. (http://www.ceaeq.gouv.qc.ca/documents/-publications/ echantillonnage.htm).

6. Chen Z., Ai, Y., Fang, C., Wang, K., Li, W., Liu, S., Li, C., Xiao, J., Huang, Z. (2014). Distribution and phytoavailability of heavy metal chemical fractions in artificial soil on rock cut slopes alongside railways. Jour. Hazar. Mat., 273: 165-173.

7. Cowan C. E, Zachara J. M., Resch C. T. (1991). Cadmium adsorption on iron oxides in the presence of alkaline 456 earth elements. Environ. Sci. Technol., 25: 437-446.

8. Daldoul, G., Souissi, R., Souissi, F., Jemmali, N., Chakroun, H. K. (2015). Assessment and mobility of heavy metals in carbonated soils contaminated by old mine tailings in North Tunisia. Jour. Afric. Ear. Sci., 22p.

9. Directive 2008/105/CE du Parlement européen et du Conseil, Journal officiel de l'Union européenne, 84-97.

10. El Azhari, A., Rhoujjati, A., EL Hachimi, M. L. (2016). Assessment of heavy metals and arsenic contamination in the sediments of the Moulouya River and the Hassan II Dam downstream of the 
abandoned mine Zeïda (High Moulouya, Morocco). Jour. Afric. Ear. Sci., 24p.

11. Helali, M. A., Oueslati, W., Zaaboub, N., Added, A., Aleya, L. (2017). Chemical speciation of $\mathrm{Fe}, \mathrm{Mn}, \mathrm{Pb}, \mathrm{Zn}, \mathrm{Cd}, \mathrm{Cu}, \mathrm{Co}, \mathrm{Ni}$ and $\mathrm{Cr}$ in the suspended particulate matter off the Mejerda River Delta (Gulf of Tunis, Tunisia). Jour. Afric. Ear. Sci.,129: 458- 468.

12. Hounkpatin, A. S., Edorh, P. A, Sèzonlin, M., Guédénon, P., Elégbédé, B., Boni, G., Dougnon, V., Montcho, S., Kéké, E., Boko, M. (2012). Pollution of aquatic ecosystems by heavy metals at Ganvié's lacustrine city (Benin). Int. Resea. Jour. Biotech. 3: 81-87.

13. Kaki, C., Guedenon, P., Kelome, N., Edorh, P. and Adechina, R. (2011). Evaluation of heavy metals pollution of Nokoue lake. Afric. Jour. Environ. Scie. Tech., 5: 255-261.

14. Laibi R. A. (2011). Evolution quaternaire et dynamique sédimentaire actuelle des cordons barrières de l'estuaire du Mono-Couffo dans le littoral du Bénin (Golfe de Guinée, Afrique de l'Ouest), Thèse de doctorat, UAC, Bénin/ ULCO, France, 307p.

15. Le Barbé, L., Ale, G., Millet, B., Texier, H.Y., Borel, Y., Gualde, R. (1993). Les ressources en eaux superficielles de la république du Bénin. Éditions de l'ORSTOM, Institut Français de Recherche Scientifique pour le Développement en Coopération, collection monographies hydrologiques $\mathrm{n}^{\circ} 11$, Paris $1993,453 \mathrm{p}$.

16. Li, H., Shi, A., Zhang, X. (2015). Particle size distribution and characteristics of heavy metals in road-deposited sediments from Beijing Olympic Park ," Jour. Environ. Sci., 32: 228- 237.

17. Moniod, F. (1973). Régime hydrologique de I'Ouémé (Dahomey). Cah. O.R.S.T.O.M., sér. Hydrol., vol. X , n 2, 13 p.

18. Okoro, H. K., Fatoki, O. S., Adekola, F. A., Ximba, B. J., Snyman, R. G., 2012. A Review of Sequential Extraction Procedures for Heavy Metals Speciation in Soil and Sediments. Open. Acces. Sci. Rep., 1(181): 1-9.

19. Olujimi, O. O., Oputu, O., Fatoki, O., Opatoyinbo, O. E., Aroyewun, O. A., Baruani, J. (2015). Heavy Metals Speciation and Human Health Risk Assessment at an Illegal Gold Mining Site in Igun, Osun State, Nigeria. Jour. Heal. Pollu. 5: 19-32.

20. Othmani, M.A., Souissi, F., Ferreira, da Silva, E., Coynel, A. (2015). Accumulation trends of metal contamination in sediments of the former Lead-Zinc mining district of Touiref (northwest Tunisia). Jour. Afric. Ear. Sci. 111: 231-243.

21. Perin, G., Craboledda, L., Lucchese, M., Cirillo, R., Dotta, L., Zanette, M. L., Orio, A. A. (1985). Heavy metal speciation in the sediments of Northern Adriatic sea - a new approach for 
environmental toxicity determination, In: T.D. Lekkas (Eds.), Heavy Metal in the Environment, no. 2, 454p.

22. Schultz, M. K., Burnett, W. C., Inn, K. G. W. (1998). Evaluation of a sequential extraction method for determining actinide fractionation in soils and sediments. Jour. Environ. Radioact. 40 (2): 155-174.

23. Sundaray, S. K., Nayak, B. B, Lin, S., Bhatta, B. (2011).Geochemical speciation and risk assessment of heavy metals in the river estuarine sediments - A case study: Mahanadi basin, India. Jour. Hazar. Mat., 186 (2011): 1837-1846

24. Tessier, A., Campbell, P. G. C., Bisson, M. (1979). Sequential extraction procedure for the speciation of particulate trace metals. Anal. Chem.51(7) 844-851.

25. Wang, J., Liu, G., Liu, H., Lam, P. K.S. (2017). Multivariate statistical evaluation of dissolved trace elements and a water quality assessment in the middle reaches of Huaihe River, Anhui, China. Sci. Tot. Environ. Article in press.

26. Youssao, A., Soclo, H. H., Bonou, C., Fayomi, B. (2011). Evaluation de la bioaccumulation du plomb dans les espèces animales marines et identification des sources de contamination métallique par une analyse multiélémentaire en métaux (Aluminum, Cadmium, Chromium, Copper, Lead) dans les eaux côtières du Bénin. Int. Jour. Biol. Chem. Sci ., 5 : 188-195.

27. Zhao, S., Feng, C., Yang, Y., Niu, J., Shen, Z. (2012). Risk assessment of sedimentary metals in the Yangtze Estuary: New evidence of the relationships between two typical index methods," Jour. Hazar. Mat., 241- 242: 164- 172.

28. Zheng, M., Feng, L., He, J., Chen, M., Zhang, J., Zhang, M., Wang, J. (2015). Delayed geochemical hazard: A tool for risk assessment of heavy metal polluted sites and case study, Jour. Hazar. Mat., $287: 197-206$.

29. Zhou, Y. (2009). Evaluation de la biodisponibilité des métaux dans les sédiments," Rapport d'étude. Agence de l'Eau Artois-Picardie et Université des Sciences et Technologies de Lille I, UMR Géosystèmes, 33 p. 